\title{
Variable range hopping in thin film with large dielectric constant
}

\author{
B.I. Shklovskii \\ Theoretical Physics Institute, University of Minnesota, Minneapolis, Minnesota 55455, USA \\ E-mail: shklovsk@physics.umn.edu
}

Received August 5, 2016, published online April 25, 2017

\begin{abstract}
In a film with large dielectric constant $\kappa$ the electric field of an electron spreads inside the film before exiting the film at large distances of order of $\kappa d$ ( $d$ is the film width). This leads to the logarithmic Coulomb repulsion between electrons and modifies the shape of the Coulomb gap in the density of localized states in a doped film. As a result the variable range hopping conductivity in such a film has a peculiar temperature dependence, where the domain of the $\ln \sigma(T) \propto\left(T_{0} / T\right)^{p}$ dependence, with the index $p \simeq 0.7$, is sandwiched between the two domains with $p=1 / 2$.

PACS: 74.81.Fa Josephson junction arrays and wire networks;

05.60.Gg Quantum transport;

74.81.Bd Granular, melt-textured, amorphous, and composite superconductors.
\end{abstract}

Keywords: Coulomb gap, thin film, hopping conductivity.

Variable range hopping (VRH) is the generic mechanism of the low-temperature transport in systems with localized electron states. When electrons repel each other via the Coulomb potential energy

$$
V(r)=\frac{e^{2}}{\kappa r},
$$

where $\kappa$ is the dielectric constant of the solid, the density of localized states $g(E)$ has the soft Coulomb gap. As a result the VRH conductivity $\sigma$ obeys the Efros-Shklovskii (ES) law $[1,2]$

$$
\sigma(T)=\sigma_{0} \exp \left[-\left(\frac{T_{0}}{T}\right)^{1 / 2}\right]
$$

both in two-dimensional (2D) and three-dimensional (3D) cases. Here the characteristic temperature

$$
T_{0}=\frac{C e^{2}}{\kappa a}
$$

(we use the energy units for the temperature $T$ ), $a$ is the localization length of electrons and $C$ is the numerical coefficient [2].

This paper deals with the situation when the Coulomb interaction between electrons has a more complicated form. We consider a thin film with the thickness $d \gg a$ and the large dielectric constant $\kappa \gg 1$. The film is surrounded by the media with much smaller dielectric constant, for example, just by the air with $\kappa_{\text {ext }}=1$.

The energy of the Coulomb repulsion of two electrons in the film was calculated exactly in Ref. 3. Here we present only asymptotic results with their physics interpretation. Let us assume that the film is defined by the surfaces $z= \pm d / 2$ and one electron is at $z=x=y=0$. Then at distances $r \ll d$ its electric field (induction) spreads isotropically. At larger distances the electric field lines are forced by the large $\kappa$ to stay inside the film, so that the field spreads along the radius $\rho=\sqrt{x^{2}+y^{2}}$ of the cylindrical coordinate system with the same $z$ axis. At $\rho \sim \kappa d$ electric field lines exit from the film and eventually spread uniformly over the whole $4 \pi$ body angle again.

Let us discuss the potential energy of repulsion of two electrons in the film. At the distance $\rho \gg \kappa d$ two electrons interact via the "external" Coulomb interaction

$$
V(\rho)=\frac{e^{2}}{\rho} .
$$

On the other hand, in intermediate range of distances $d \ll \rho \ll \kappa d$

$$
V(\rho)=2 \varepsilon_{d} \ln \left(\frac{\kappa d}{\rho}\right)
$$


where $\varepsilon_{d}=e^{2} / \kappa d$. Finally at even smaller distances $\rho \ll d$ we arrive at the "internal" Coulomb interaction

$$
V(r)=\frac{e^{2}}{\kappa r}+2 \varepsilon_{d} \ln \kappa,
$$

which differs from Eq. (1) by the logarithmically large energy accumulated when the second electron is moved from infinity to $\rho=d$. The resulting potential energy $V(\rho)$ is plotted as a function of $\rho$ in Fig. 1 in all three ranges.

Let us assume that the film is uniformly doped by a concentration of donors $N_{D}$ and compensated by smaller concentration of acceptors so that low-temperature transport is due to VRH on donors. How does the two-dimensional electrostatics affect ES law?

This question was first addressed in Ref. 4 for another object, a weakly disordered two-dimensional gas, for example, in a silicon inversion layer. In this case, according to Ref. 5 localized states have an exponentially large localization length. Large localized states play the dual role in this theory. First, VRH conductivity is related to long distance hopping between those of them which are close to the Fermi level. Second, all other large localized states according to Ref. 4 contribute to the large effective dielectric constant which keeps the electric lines of a charge in the plane of the two-dimensional gas. In order to calculate VRH conductivity the authors of Ref. 4 used an intuitive shortcut avoiding discussion of the Coulomb gap. The authors found two ranges of the temperature dependence of the VRH conductivity $\sigma(T)$. At very low temperatures where the characteristic length of the hop $r_{h}$ is much larger than $\kappa d$ they arrived at ES law with $\kappa=1$ and at higher temperature range where $d \ll r_{h} \ll \kappa d$ they obtained activated conductivity.

In this paper, we calculate the VRH conductivity in the framework of our simpler model where large $\kappa$ is of

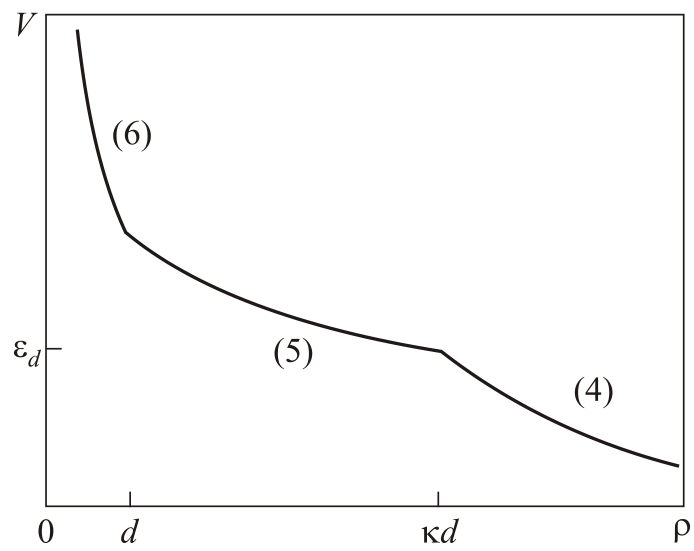

Fig. 1. A schematic plot of the potential energy of repulsion of two charges located inside the film with a large dielectric constant as a function of the distance $\rho$ between electrons. Equation numbers describing different segments of this plot are shown next to each segment. the lattice origin or is a result of close three-dimensional metal-insulator transition. In this way we avoid the controversial subject of the $2 \mathrm{D}$ metal-insulator transition [6]. We follow the "orthodox" ES logic [1,2] deriving first the Coulomb gap and then the conductivity. Let us first formulate our results moving from high to lower temperatures.

At high temperatures $T \gg \varepsilon_{d}$ a/d the VRH hopping conductivity is determined by the "internal" ES law

$$
\sigma(T)=\sigma_{0} \exp \left[-\left(\frac{T_{0}}{T}\right)^{1 / 2}-\frac{\varepsilon_{d} \ln \kappa}{T}\right],
$$

which at high enough temperatures coincides with Eq. (2).

It is followed by the range of intermediate temperatures

$$
\frac{\varepsilon_{d} a}{\kappa d} \ll T \ll \frac{\varepsilon_{d} a}{d}
$$

with "activated" VRH conductivity

$$
\sigma(T)=\sigma_{0} \exp \left[-\frac{T_{1}(T)}{T}\right],
$$

where the "activation energy"

$$
T_{1}(T)=\varepsilon_{d} \ln \left(\frac{T}{\varepsilon_{d}} \frac{\kappa d}{a}\right),
$$

of the intermediate regime is weakly temperature dependent. If one approximates Eq. (9) as

$$
\sigma(T)=\sigma_{0} \exp \left[-\left(\frac{T_{p}}{T}\right)^{p}\right],
$$

the power

$$
p=1-\frac{1}{\ln \left(T \kappa d / \varepsilon_{d} a\right)} .
$$

Close to $T=\varepsilon_{d} a / d$ we get $p=1-1 / \ln \kappa$. For example, at $\kappa=40$ one gets $p=0.7$.

At even smaller temperatures $T \ll \varepsilon_{d} a / \kappa d$ we arrive at the "external" ES law

$$
\sigma(T)=\sigma_{0} \exp \left[-\left(\frac{T_{0}^{\text {ext }}}{T}\right)^{1 / 2}\right]
$$

where $T_{0}^{\mathrm{ext}}=C e^{2} / a$. Thus, the "activated" VRH conductivity (9) is sandwiched between the two different ES regimes of $\sigma(T)$, the "internal" one, Eq. (7), at the high-temperature side, and the "external" one, Eq. (13), on the lowtemperature side. The two low-temperature regimes (9) and (13) are in agreement with Ref. 4. The new hightemperature "internal" ES regime exists only if $d \gg a$.

The experimental literature on the VRH conductivity in thin films is controversial (see Refs. 7, 8 and references therein). How large is the film dielectric constant and how important is contribution of large localized states [4] in most 
of cases is not clear. On the insulating side of the superconductor-insulator transition in ultrathin quench-condensed $\mathrm{Ag}, \mathrm{Bi}, \mathrm{Pb}$ and $\mathrm{Pd}$ films [7] $\mathrm{VRH}$ data agree with Eq. (11) with $p=2 / 3$. On the other hand, for relatively thick TiN films [8] the crossover from $p \simeq 1 / 2$ to $p \simeq 1$ is observed with the decreasing temperature. Finding an explanation for this crossover is challenging [8] because one would normally expect that the ES law emerges at the lowtemperature limit. Our theory shows that in relatively thick film with the decreasing temperature one can see the crossover from the "internal" ES law to the activated transport. This may explain results of Ref. 8 .

In order to simulate a film with a large dielectric constant one can make a two-dimensional array of isolated metallic islands overhanging each other $[9,10]$. Although these arrays were originally designed as arrays of Josephson junctions they perfectly simulate a large dielectric constant in the normal state. Indeed, such array keeps electric lines in its plane if the capacitance between two islands is larger than the capacitance of each island to the ground. As a result such a normal array in presence of some disorder should show activated VRH discussed above.

Before switching to the derivation of our results we would like to dwell on the related theoretical paper [11] which deals with the VRH transport of point like vortexes responsible for the low-temperature resistance of a superconductor film in the external magnetic film. Two vortexes interact via the logarithmic potential at small distances, while again at large distances their interaction follows the "external" Coulomb potential [12], so that one could expect to see the two low-temperature ranges discussed above, the "activated" regime and the "external" ES law. However, Ref. 11 argues that ES approach is not valid in this case because "logarithmic interaction grows without bound with particle separation" and, therefore, "single-particle energies can not be defined" so that "multi-vortex hopping dominates the above single-particle effects". The multi-particle estimate [11] leads to Eq. (11) with $2 / 3<p<4 / 5$. This is close to what we got for $p$ at $\kappa=40$.

In contrary to the above statements of Ref. 11 Fig. 1 clearly shows that the repulsion energy vanishes at infinity similarly to the standard Coulomb potential (4). Thus, there is no problem to introduce a single particle energy for a system of localized electrons interacting with the pairwise potential $V(\rho)$. We can proceed with the ES argument and study the new shape of the Coulomb gap in the density of states (DOS) of single-particle excitations and eventually the VRH conductivity. We return to the discussion of the role of multi-particle processes in the end of this paper.

Below we calculate the zero temperature DOS $g(\varepsilon)$ following Refs. 1, 2, 13. In the ground state of the system we define the single electron energy $\varepsilon_{i}$ of an occupied donor $i$ as the energy necessary to extract an electron from this state to the state with the energy right at the Fermi level at infinity. The single-electron energy $\varepsilon_{j}$ of an empty in the ground state donor $j$ is defined as the energy necessary to bring to it an electron from a state at infinity with the energy right at the Fermi level $\varepsilon_{F}$. By the definition of the ground state $\varepsilon_{j}>\varepsilon_{F}>\varepsilon_{i}$. Another stronger stability condition can be formulated for each pair of occupied and empty states as follows

$$
\varepsilon_{j}-\varepsilon_{i}-V\left(r_{i j}\right)>0
$$

Here $V\left(r_{i j}\right)$ is the repulsion energy of two electrons on sites $i$ and $j$ and $-V\left(r_{i j}\right)$ is the Coulomb energy of attraction of the electron which moved to the site $j$ by the hole it has left at the site $i$. In other words, this term describes the exciton effect. Eq. (14) requires that any two states close in energy to $\varepsilon_{F}$ should be far enough in the space. This limits the density of states (DOS) close to the Fermi level. For the Coulomb potential the result for the DOS is known and we do not repeat derivations from $[1,2,13]$, but list the results.

The "external" Coulomb potential (4) limits two-dimensional density of states at the level

$$
g(\varepsilon)=\frac{2}{\pi} \frac{\varepsilon}{e^{4}}
$$

For the "internal" Coulomb potential (6) we get

$$
g(\varepsilon)=\frac{3}{\pi} \frac{\kappa^{3} \varepsilon^{2} d}{e^{6}}
$$

where the factor $d$ converts the three-dimensional DOS to the two-dimensional one. Apparently the asymptote (15) is valid at large distances, i.e., at small energies $\varepsilon$ and the asymptote (15) describes small lengths or large energies. Let us now derive the DOS for the intermediate range of distances and energies. To this end we have to calculate the number of states in the band of the width $2 \varepsilon$ around $\varepsilon_{F}$. Using Eqs. (14) and (5) we get $\varepsilon_{d} \ln (\kappa d / \rho)<\varepsilon$. This means that in this energy band there is no more than one state in the disc with radius

$$
\rho(\varepsilon)=\kappa d \exp \left[-\frac{\varepsilon}{\varepsilon_{d}}\right] .
$$

This leads to the following estimate of the DOS for $\varepsilon_{d} \ll \varepsilon \ll \varepsilon_{d} \ln \kappa$ :

$$
g(\varepsilon) \sim \frac{1}{\rho(\varepsilon)^{2} \varepsilon}=\frac{1}{(d \kappa)^{2} \varepsilon} \exp \left[\frac{2 \varepsilon}{\varepsilon_{d}}\right] .
$$

DOS (18) matches DOS (15) at $\varepsilon=\varepsilon_{d}$ and Eq. (16) at $\varepsilon=\varepsilon_{d} \ln \kappa$ (see Fig. 2). At large energies the parabolic range of the Coulomb gap, Eq. (16), is limited by the total width of the impurity band $\varepsilon_{\max }=e^{2} N_{D}^{1 / 3} / \kappa+\varepsilon_{d} \ln \kappa$. At $\varepsilon \gg \varepsilon_{\max }$ the DOS decreases (see the dashed line in Fig. 2) similarly to the three-dimensional DOS of the classical impurity band (see Ch. 14 of Ref. 2). 




Fig. 2. Schematic plot of the DOS of localized electrons limited by Coulomb interaction of electrons as a function of energy. Equation numbers describing different segments of this plot are shown next to each segment. Dashed line shows beginning of the DOS decline at large energies.

Now we can apply the calculated above DOS in order to estimate the exponential term of the VRH conductivity of the film at different temperatures. This estimate closely follows the original Mott's approach [14]. We define an energy band around the Fermi level and estimate contribution of this band to the VRH conductivity. Then we can optimize result with respect of the band width. This calculations are quite straightforward and we will not go through them here. Results are already formulated in the beginning of our paper.

Let us return to the multi-electron effects on ES law. For the standard Coulomb potential (1) they were studied in Refs. 2, 13, 15, 16. It was shown that they may change only the coefficient $C$ in Eq. (2). Here we only briefly remind what was done. In 3D case a small energy singleelectron excitation strongly interacts with dipole moments of surrounding compact electron-hole excitations forming together with them a composite charged multi-electron excitation, the electronic polaron. Polarons being charged particles obey stability criterion Eq. (14), have the Coulomb gap and lead to ES law. The only difference is that coefficient $C$ can be somewhat larger because every hop of a single-electron excitation in 3D is accompanied by the tunnelling depolarization of many polaron pairs. A simple estimate showed that the total length of these small hops is of the order of the length of the main long ES VRH hop. This gives no more than the factor 2 in the expression for $C$. In 2D number of dipole excitations in the polaron atmosphere is of order one and polaron effects provide only small corrections to $C$.

We estimated similar effects in the framework of the complex potential of this paper (Eqs. (4)-(6)). It turns out that in the low-temperature range polaron effects provide only small corrections to $C$ in Eq. (13). On the other hand, for the two higher temperature ranges (Eqs. (9) and (2)) the situation is similar to the 3D case studied in Refs. 2, 13, 16.
Namely, at these temperatures multi-electron effects may add a numerical coefficient in the exponential. For Eq. (9) this means that $T_{1}$ may become twice larger. Thus, multielectron effects do not change the power $p$ in Eq. (11). This conclusion is in disagreement with Ref. 11 which assumes that even in 2D the total length of small hops is much larger than the ES hop and, therefore, overestimates importance of the multi-electron effects.

Above we discussed only the ohmic transport in a weak electric field. If the electric field is so strong that $e E a / 2 \gg T$ one can replace $T$ by the effective VRH temperature $e E a / 2$ in Eqs. (2), (9), (11) and (13) to obtain non-ohmic current-voltage characteristics [17] (see also [4,18]). For the intermediate activated regime we then arrive at the current-voltage characteristics $J \propto J_{0} \exp \left(-E_{0} / E\right)^{p}$, where $p$ is close to unity. This result is in agreement with the earlier theory [19] of the VRH transport of pinned vortexes in superconductors under the influence of a strong current.

Until now we dealt with a film. Now let us briefly discuss the similar physics in a long cylindrical nano-wire or nano-rod with radius $d$ made from a semiconductor with a large dielectric constant $\kappa \gg 1$, for simplicity, in the air environment. Here again the electric field of an electron at distances $r \ll d$ spreads isotropically, then stays inside nano-rod for a distance $x<\xi=d \kappa^{1 / 2}$ along the cylinder axis, and then leaks from the cylinder and eventually spreads isotropically in the air at large enough $r$. Thus, the potential energy of repulsion of two electrons again changes from the very short range "internal" Coulomb interaction (4) to the very long range "external" Coulomb interaction (1) with the large intermediate range of $x$, where interaction has the one-dimensional character. The interaction in this range was studied [20,21] for an ion channel in a lipid membrane, where the cylindrical pore with radius $d$ is filled by water with $\kappa=81$ and is surrounded by lipids with $\kappa=2$. Translated to our problem the potential energy of two electrons located at the nano-rod axis at the intermediate distance $x$ from each other is well approximated by

$$
V(x)=e E_{0} \xi[\exp (-x / \xi)-1]
$$

where $E_{0}=2 e / \kappa d^{2}$ (see Sec. VIII of Ref. 21). Using this potential for repulsion of two electrons in the nano-rod and following the Coulomb gap based derivation similar to one used above for a film (or the shortcut approach of Ref. 4) one can calculate the temperature dependence of the VRH conductivity of the nano-rod in the intermediate temperature range. The result is the strict activation regime with the temperature independent activation energy $T_{a}=e E_{0} \xi$. It is, of course, sandwiched between the two ES laws, the "internal" one on the high-temperature side and the "external" one Eq. (13) on the low-temperature side.

I am grateful to T. Baturina, A.M. Goldman, A. Kamenev, D.E. Khmelnitskii and A.I. Larkin for useful discussions. 
1. A.L. Efros and B. Shklovskii, J. Phys. C 8, L49 (1975).

2. B.I. Shklovskii and A.L. Efros, Electronic Properties of Doped Semiconductors, Springer, New York (1984). This book is available from http://www.tpi.umn.edu/shklovskii/

3. L.V. Keldysh, Sov. Phys. JETP Lett. 30, 245 (1979).

4. A.I. Larkin and D.E. Khmelnitskii, Sov. Phys. JETP 56, 647 (1982).

5. E. Abrahams, P.W. Anderson, D.C. Liccardello, and T.V. Ramakrishnan, Phys. Rev. Lett. 42, 673 (1979).

6. E. Abrahams, S.V. Kravchenko, and M.P. Sarachik, Rev. Mod. Phys. 73, 251 (2001).

7. N. Markovic, C. Christiansen, D.E. Grupp, A.M. Mack, G. Martinez-Arizala, and A.M. Goldman, Phys. Rev. B 62, 2195 (2000).

8. T.I. Baturina, A.Yu. Mironov, V.M. Vinokur, M.R. Baklanov, and C. Strunk, Phys. Rev. Lett. 99, 257003 (2007).

9. E. Chow, P. Delsing, and D.B. Haviland, Phys. Rev. Lett. 81, 204 (1998).

10. M.V. Fistul, V.M. Vinokur, and T.I. Baturina, Phys. Rev. Lett. 100, 086805 (2008).
11. M.P.A. Fisher, T.A. Tokuasu, and A.P. Young, Phys. Rev. Lett. 66, 2931 (1991).

12. J. Pearl, Appl. Phys. Lett. 5, 65 (1964); P.G. De Gennes, Superconductivity of Metalls and Alloys, W.A. Benjamin, New York-Amterdam (1966).

13. A.L. Efros and B.I. Shklovskii, in: Electron-Electron Interaction in Disordered Systems, A.L. Efros and M. Pollak (eds.), North-Holland-Amsterdam (1985).

14. N.F. Mott, J. Non-Cryst. Solids 1, 1 (1968).

15. A.L. Efros, J. Phys. C 9, 2021 (1976).

16. S.D. Baranovskii, B.I. Shklovskii, and A.L. Efros, Sov. Phys. JETP 51, 199 (1980).

17. B.I. Shklovskii, Sov. Phys.-Semicond. 6, 1964 (1973).

18. S. Marianer and B.I. Shkiovskii, Phys. Rev. B 46, 13100 (1992).

19. D.R. Nelson and V.M. Vinokur, Phys. Rev. B 48, 13060 (1993).

20. A. Kamenev, J. Zhang, A.I. Larkin, and B.I. Shklovskii, Physica A 359, 129 (2006).

21. J. Zhang, A. Kamenev, and B.I. Shklovskii, Phys. Rev. E 73, 051205 (2006). 